\title{
Comparison of nonmicroprocessor knee mechanism versus C-Leg on Prosthesis Evaluation Questionnaire, stumbles, falls, walking tests, stair descent, and knee preference
}

\author{
Jason T. Kahle, CPO, LPO; ${ }^{1}$ M. Jason Highsmith, DPT, CP; ${ }^{1-2 *}$ Sandra L. Hubbard, PhD, OTR/L, ATP ${ }^{3-4}$ \\ ${ }^{1}$ Westcoast Brace and Limb, Tampa, FL; ${ }^{2}$ School of Physical Therapy \& Rehabilitation Sciences, University of South \\ Florida, Tampa, FL; ${ }^{3}$ Rehabilitation Outcomes Research Center of Excellence, North Florida/South Georgia Veterans \\ Health System, Gainesville, FL; ${ }^{4}$ Department of Occupational Therapy, University of Florida, Gainesville, FL
}

\begin{abstract}
This study compared subjects' performance with a nonmicroprocessor knee mechanism (NMKM) versus a C-Leg on nine clinically repeatable evaluative measures. We recorded data on subjects' performance while they used an accommodated NMKM and, following a 90-day accommodation period, the C-Leg in a convenience sample of 19 transfemoral (TF) amputees (mean age $51+/-19$ ) from an outpatient prosthetic clinic. We found that use of the C-Leg improved function in all outcomes: (1) Prosthesis Evaluation Questionnaire scores increased 20\% ( $p=0.007)$, (2) stumbles decreased 59\% ( $p=$ $0.006)$, (3) falls decreased $64 \%(p=0.03)$, (4) $75 \mathrm{~m}$ selfselected walking speed on even terrain improved 15\% ( $p=$ 0.03), (5) $75 \mathrm{~m}$ fastest possible walking speed (FPWS) on even terrain improved $12 \%$ ( $p=0.005)$, (6) $38 \mathrm{~m}$ FPWS on uneven terrain improved 21\% ( $p<0.001)$, (7) $6 \mathrm{~m}$ FPWS on even terrain improved $17 \%(p=0.001)$, (8) Montreal Rehabilitation Performance Profile Performance Composite Scores for stair descent increased for 12 subjects, and (9) the C-Leg was preferred over the NMKM by 14 subjects. Four limited community ambulators (Medicare Functional Classification Level [MFCL] K2) increased their ambulatory functional level to unlimited community ambulation (MFCL K3). Objective evaluative clinical measures are vital for justifying the medical necessity of knee mechanisms for TF amputees. Use of the C-Leg improves performance and quality of life and can increase MFCL and community ambulation level.
\end{abstract}

Key words: amputee, C-Leg, falls, microprocessor knee mechanism, nonmicroprocessor knee mechanism, prosthetic knee mechanism, rehabilitation, stairs, stumbles, transfemoral, transfemoral amputation, walking.

\section{INTRODUCTION}

Amputees seek prostheses that meet their functional needs to the fullest extent possible. For transfemoral (TF) amputees, a prosthetic knee mechanism is required. However, the number and type of available prosthetic knees from which to choose can be overwhelming. Selection and prescription were complex tasks even before the introduction of microprocessor knee mechanisms (MKMs). Select MKMs analyze a patient's gait at a rapid rate (501,000 times/second) and translate the information into the functional need required at that instant. If a patient begins to stumble or fall, the microprocessor can sense the irregularity and decrease the rate of knee flexion. This adjustment

\footnotetext{
Abbreviations: CMS $=$ Centers for Medicare and Medicaid Services, FPWS $=$ fastest possible walking speed, MFCL = Medicare Functional Classification Level, MKM = microprocessor knee mechanism, MRPP = Montreal Rehabilitation Performance Profile, NMKM = nonmicroprocessor knee mechanism, PCS = Performance Composite Score, PEQ = Prosthesis Evaluation Questionnaire, PT = physical therapy, SSWS = self-selected walking speed, TF $=$ transfemoral.

*Address all correspondence to M. Jason Highsmith, DPT, CP; School of Physical Therapy \& Rehabilitation Sciences, University of South Florida, 12901 Bruce B. Downs Blvd, MDC 77, Tampa, FL 33612-4766; 813-974-3806; fax: 813974-8915. Email: mhighsmi@health.usf.edu

DOI: 10.1682/JRRD.2007.04.0054
} 
allows the patient time to recover by increasing the stability of the prosthetic knee. This feature of an MKM is known as stumble recovery. If a patient is descending stairs or a slope, the microprocessor will quickly sense this change and slowly and safely allow the patient to descend the stairs or slope by decelerating the rate of knee flexion. When a patient changes walking speed, the microprocessor can quickly accelerate or decelerate the rate of flexion and extension to accommodate for the variable cadence of the user. In short, the knee's microfunction is monitored and controlled by the microprocessor, not the patient. MKM designers claim that these features lead to a safer, more efficient gait.

When new prosthetic components are introduced, clinicians and researchers must ask questions such as: Who is a candidate for the new component? What qualities will result in rejection/acceptance? Will this component afford functional gain? Will the latest technological prosthetic componentry, e.g., the C-Leg ${ }^{\circledR}$ (Otto Bock; Minneapolis, Minnesota) MKM, garner significant functional gain? Will the gains be perceived, observable, or both? Will the gains be realized most in stability, gait, or stair performance? In which subgroup of the larger TF amputee population will benefits be most appreciable?

In order to answer some of the numerous questions that surround MKM candidacy, we must select ecologically comparable tests that apply to the realistic and practical circumstances faced daily by users. Similarly, to ensure transfer and application in the clinical setting, we must select tests and measures that are brief, cost-effective, and relevant. To carry scientific merit, tests must be substantiated in the literature and have successfully passed rigorous validity and reliability benchmarks. Several outcomes and tests are available for evaluating prosthetic knee function. Selecting specific outcomes of interest significantly narrows test selection.

A battery of repeatable and easily administered tests that a practitioner could use in the clinical setting to evaluate a patient's perceived and observable performance on a prosthetic knee mechanism is imperative for comparing knee mechanisms. Such a protocol could prove vital for (1) clinically determining a patient's functional performance and (2) determining and/or justifying medical necessity, patient need, and patient preference for one component versus another.

We selected the following outcome categories to determine optimum knee function: perceived performance and knee preference, stumbles and falls, clinically relevant walking tests, and stair descent. We briefly discuss each outcome category in the following sections.

\section{Perceived Performance and Knee Preference}

The significance of patient input into the prescription, component/knee selection, and fabrication of a prosthesis and its influence on successful outcomes is well recognized [1-3]. Boone and Coleman state that "even with optimal biomechanical action, the user could have [a] very strong negative reaction due to other matters of importance to them" [3, p. P68]. Similarly, patients often desire to please and, when asked directed questions, provide subjective answers that they believe providers and/ or researchers want to hear. Another confounding issue with subjective data collection is the well-documented placebo effect. This effect is much more difficult to deal with in some types of studies than in others. For example, blinding patients to the fact that they were previously using a Mauch SNS ${ }^{\circledR}$ (Ossur; Reykjavík, Iceland) but are now using a C-Leg is very difficult. The fact that people are using these high-tech components may be enough for them to believe that they are experiencing enhanced function, even though the component may or may not be most appropriate in their case.

In an attempt to circumvent some of the pitfalls associated with subjective data collection yet still include subjective data because of the value of capturing participant input and preference, we should consider two methods. The first method is to use a population-specific, validated, and reliable instrument. The Prosthesis Evaluation Questionnaire (PEQ) evaluates (1) prosthesis function and (2) prosthesis-related quality of life and is population specific as well as valid and reliable [2]. The second method to consider is directly asking participants their component preference. Hafner states that studies can be complemented by this question, the answer to which can be used to strengthen or refute findings of a study [1]. Asking which component is preferred, particularly if the component is to be kept by the participant, might very well discourage an answer the investigator wishes to hear and identify the actual component the participant wishes to take home and use long-term.

\section{Stumbles and Falls}

Falling is pervasive among amputees. More than 60 percent of TF amputees have experienced at least one fall. Pain, multiple residual limbs, and prosthetic problems as well as the need to concentrate while walking are 
risk factors for increased falls [4]. Numerous methods have been used to track and study stumbles, falls, and associated injuries. Methods include subject recall of events, provision of a journal where subjects can log events, interval mailing of postcards, calendar marking, and medical record review. Mackenzie et al. used calendar marking as the gold standard against which to compare subject recall [5]. Investigators point out that even this gold standard has limitations and is subject to significant misreporting. For example, the accuracy of calendar marking is subject to the subject's level of compliance and diligence in record keeping. If participants are not diligent in record keeping, marking a calendar becomes little more than a journal of sporadic recall. All methods listed, except recall, have the drawback of increasing burden on subjects. The use of recall, however, has several of its own limitations. For instance, recall is subject to the proximity, saliency, and attachment of an event. It is also limited by the extent of memory decay over time, true under- or overestimation, and intervention bias [5-7]. Despite these limitations, the inclusion of self-reported stumbles and falls is crucial information because the subject is often the sole witness to the event. Jenkins et al. reported that injury recall for periods $>2$ months is subject to significant underestimation and that, because of memory decay, recall of periods of $\geq 12$ months should be accompanied by validation strategies that ascertain and correct for the level of decay [6].

In this study, we chose to use subject recall of stumbles and falls as opposed to one of the other methods for the following reasons: (1) the recall period was relatively short, (2) the subjects were likely to be free from prospective comorbidities that affect memory (e.g., cerebral vascular accident, dementia, traumatic brain injury), (3) the benefit of associating recall with the salient event of a knee-mechanism exchange, and (4) the possibility inherent in the other methods of excessive subject burden that could lead to attrition.

\section{Walking Tests}

Several studies have compared various outcomes associated with use of MKMs versus nonmicroprocessor knee mechanisms (NMKMs) [8-15]. In a within-subject study of $12 \mathrm{TF}$ amputees, Schmalz et al. reported a 6 percent reduction in oxygen consumption rate (milliliter/ kilogram/minute) at slower walking speeds for the C-Leg versus an NMKM [8]. They did not find a statistically significant difference at faster walking speeds, and their methodology and analyses have both been challenged
[9]. Like Schmalz et al., Johansson et al. reported a similarly reduced, though not statistically significant, metabolic gait cost via oxygen consumption rate when subjects walked with a C-Leg versus a Mauch SNS [10]. Orendurff et al. found that subjects using the C-Leg had a lower net oxygen cost (milliliter/kilogram/meter) compared with those using the Mauch SNS in a sample of eight TF amputees, but again, the difference failed to reach statistical significance [9]. Highly technical analysis of expired air exceeded the parameters of this study, i.e., the focus on a clinically relevant battery of tests. Orendurff et al. also reported that subjects' self-selected walking speed (SSWS) was faster when using the C-Leg, though oxygen cost was not increased. This result suggests greater SSWS efficiency. Orendurff et al. concluded that before an optimal prosthetic limb can be prescribed to improve TF amputees' walking efficiency, we need an assessment that is more objective, valid, and comprehensive than metabolic data [9]; SSWS is a more clinically friendly and available performance measure. That is, distance-based ambulatory tests were deemed more clinically usable than metabolic data and were therefore used in this study. We collected data for four distance-based walking tests: $75 \mathrm{~m}$ SSWS on even terrain, $75 \mathrm{~m}$ fastest possible walking speed (FPWS) on even terrain, $38 \mathrm{~m}$ FPWS on uneven terrain, and $6 \mathrm{~m}$ FPWS on even terrain.

\section{Stair Descent}

The ability to successfully negotiate stairs is an important factor for functional independence [16]. In the absence of pathology, stairs are negotiated with greater confidence and fewer adaptive strategies [17-18]. The amputee population should be included in the focus on what has been described for nondisabled individuals as "functional fitness," which includes stair ascent and descent among other things. Furthermore, the inability to walk down stairs has been associated with a catastrophic decline in mobility [19]. Van Iersal et al. conducted a systematic literature review to identify the way functional assessment scales evaluate stair negotiation [20]. Of the 92 identified scales, 43 have an item on stair negotiation, emphasizing the importance of including stair descent in a functional assessment. A claimed benefit of some prosthetic knee mechanisms is that subjects can descend stairs in a step-over-step pattern, whereas for other mechanisms, leading descent with the prosthesis is recommended. We selected the Montreal Rehabilitation Performance Profile (MRPP) to evaluate stair descent because it considers a step-over-step pattern, cueing, timing, and other variables 
pertinent to the task of interest and also because no instruments exist that have been validated for TF amputees relative to stair descent [21].

This study compared subjects' performance using a NMKM versus a C-Leg on the previously described outcomes. We hypothesized that persons using a C-Leg would (1) rate higher levels of perceived prosthesis function and prosthesis-related quality of life, (2) self-report fewer stumbles and falls, (3) cover a given distance in less time (faster walking speed), (4) descend stairs in a step-over-step pattern with fewer errors, and (5) ultimately prefer the C-Leg over their previously accommodated NMKM.

\section{METHODS}

\section{Design and Participants}

Twenty-one TF amputees of varied etiology gave their informed consent to participate in this study (Table 1). The study protocol was approved by an ethics review committee. We used a pre- to posttest (A-B) design because it is the closest representation of clinical prosthetic practice, e.g., when patients transition from a preparatory to a definitive knee mechanism or from a definitive NMKM to an MKM.

Individually by assessment and then by consensus, a certified licensed prosthetist, a licensed physical therapist, and the subject's physician determined each subject's reasonable ambulatory potential and rated all the candidates according to Medicare Functional Classification Level (MFCL). Criteria for these levels included the subject's history and current medical and ambulatory status as well as the subject's desire and potential to ambulate.

Inclusion criteria were (1) TF or knee-disarticulation amputation, (2) self-reported or known use of existing NMKM for at least 90 days before enrolling in the study, (3) at $\geq 1$ episode of physical therapy (PT) intervention before enrolling, (4) ability to ambulate in the community with or without an assistive device but with no human support, (5) ability to descend stairs with no human support, and (6) willingness to continue NMKM use for 90 days followed by C-Leg use for 90 days. MFCLs were established for each subject at pretesting and again at posttesting; all subjects were required to maintain at least a K2 MFCL throughout the study.

Table 1.

Subject demographic information $(N=19$, mean \pm standard deviation age $=51 \pm 19 \mathrm{yr})$.

\begin{tabular}{|c|c|c|c|c|c|c|c|c|}
\hline Subject & Age (yr) & Race & Side & Etiology & Amputation Year & Height (cm) & Weight (kg) & Employed \\
\hline $\mathrm{A}$ & 59 & $\mathrm{~W}$ & $\mathrm{~L}$ & PVD & 2002 & 180 & 91.2 & $\mathrm{Y}$ \\
\hline B & 62 & W & $\mathrm{R}$ & Trauma & 1971 & 173 & 69.4 & $\mathrm{~N}$ \\
\hline $\mathrm{C}$ & 54 & $\mathrm{~W}$ & $\mathrm{~L}$ & $\mathrm{DM}$ & 2003 & 173 & 113.4 & $\mathrm{~N}$ \\
\hline $\mathrm{D}$ & 27 & W & $\mathrm{R}$ & Cong & - & 150 & 53.5 & $\mathrm{Y}$ \\
\hline $\mathrm{E}$ & 56 & W & $\mathrm{L}$ & $\mathrm{DM}$ & 2002 & 163 & 57.2 & $\mathrm{Y}$ \\
\hline $\mathrm{F}$ & 77 & $\mathrm{H}$ & $\mathrm{R}$ & Trauma & 1996 & 168 & 56.2 & $\mathrm{Y}$ \\
\hline G & 29 & $\mathrm{H}$ & $\mathrm{R}$ & Cong & - & 142 & 46.3 & $\mathrm{Y}$ \\
\hline $\mathrm{H}$ & 42 & A & $\mathrm{L}$ & Trauma & 2002 & 170 & 64.4 & $\mathrm{~N}$ \\
\hline I & 52 & $\mathrm{~W}$ & $\mathrm{R}$ & Cong & - & 160 & 59.0 & $\mathrm{~N}$ \\
\hline $\mathrm{J}$ & 63 & W & $\mathrm{L}$ & Sarcoma & 2000 & 160 & 52.6 & $\mathrm{Y}$ \\
\hline K & 41 & W & $\mathrm{R}$ & Trauma & 1992 & 170 & 55.3 & $\mathrm{Y}$ \\
\hline $\mathrm{L}$ & 57 & W & $\mathrm{L}$ & $\mathrm{DM}$ & 2001 & 170 & 67.1 & $\mathrm{~N}$ \\
\hline M & 31 & $\mathrm{~W}$ & $\mathrm{R}$ & Trauma & 2002 & 165 & 79.4 & $\mathrm{Y}$ \\
\hline $\mathrm{N}$ & 83 & W & $\mathrm{L}$ & PVD & 2004 & 183 & 65.8 & $\mathrm{~N}$ \\
\hline $\mathrm{O}$ & 83 & W & $\mathrm{L}$ & PVD & 2004 & 168 & 56.7 & $\mathrm{~N}$ \\
\hline$P$ & 28 & W & $\mathrm{R}$ & Trauma & 2004 & 188 & 98.0 & S \\
\hline $\mathrm{Q}$ & 37 & $\mathrm{~W}$ & $\mathrm{R}$ & Trauma & 1988 & 168 & 59.4 & $\mathrm{Y}$ \\
\hline $\mathrm{R}$ & 72 & W & $\mathrm{L}$ & $\mathrm{DM}$ & 2001 & 163 & 56.7 & $\mathrm{~N}$ \\
\hline $\mathrm{S}$ & 22 & W & $\mathrm{L}$ & Cong & - & 160 & 61.2 & S \\
\hline
\end{tabular}


Subjects had to be willing to comply with follow-up visits (i.e., for alignment, adjustment, and retesting) and had to be medically cleared for ambulation with a prosthesis. Exclusion criteria were the inability to satisfy any or all inclusion criteria.

\section{Evaluative Measures}

We selected nine evaluative measures to compare performance outcomes between the accommodated NMKM and the C-Leg. They were administered in the following order: (1) PEQ [2], (2) self-reported number of falls, (3) self-reported number of stumbles, (4) $75 \mathrm{~m}$ SSWS on even terrain [22-23], (5) 75 m FPWS on even terrain [22-25], (6) $38 \mathrm{~m}$ FPWS on uneven terrain [2225], (7) $6 \mathrm{~m}$ FPWS on even terrain [22-38], (8) stair descent test (MRPP) [21], and (9) knee preference [1].

\section{Prosthesis Evaluation Questionnaire}

The PEQ measures subjective prosthesis function and prosthesis-related quality of life. We chose this outcome measure based on the assumption that even if observable differences were found between the C-Leg and NMKM, subjects may still reject a component for nonperformance issues, e.g., lack of perceived improvement. The PEQ has been determined to be reliable and valid with lower-limb amputees [2].

\section{Stumbles and Falls}

Stumbles and falls were tracked by self-report with the following questions: for stumbles, "How many times in the last 60 days did any event occur in which you felt your prosthesis became temporarily unstable and you felt you were at risk of falling but did not?” and for falls, "How many times did an event occur that caused you to fall to the ground?” Using these questions, we quantified subjects' number of stumbles and falls during the last 60 days as opposed to the 90 -day intervention period. We believed this data would better represent how many events might realistically occur well into accommodation based on the assumption that a disproportionate number of stumbles and falls are likely to occur in the first 30 days of use of any new component, which would create an unfair comparison between the newly fitted C-Leg and the well-accommodated NMKM.

\section{Self-Selected and Fastest Possible Walking Speeds: $75 \mathrm{~m}$ on Even Terrain}

The SSWS and FPWS tests were designed for use with this study based on clinically useful walking tests in nonamputee populations described in the literature [2225]. Differing scores on the SSWS and FPWS objectively demonstrate subjects' ability to vary cadence. Variable cadence is valuable to the amputee, particularly the geriatric patient, for two reasons. First, the ability to vary walking speed is a reimbursement criteria used by the Centers for Medicare and Medicaid Services (CMS) [39]. Second, persons with a more normal walking speed are at less risk for falling [22-23].

The dilemma with the SSWS and FPWS function tests is selecting an appropriate distance. No one test designates what distance should be used with specific population(s). According to the initial Walter Reed C-Leg candidacy protocol, users had to be able to walk more than 400 yards daily to qualify for a C-Leg [40]. While this 400-yard distance is appropriate for a prosthetic user who is a warrior athlete, it exceeds the demands of basic ambulation and is too rigorous for the majority of the dysvascular TF amputee population. Horemans et al. established reliable measures of "preferred" (self-selected) and maximal (fastest possible) walking speeds in individuals with postpoliomyelitis syndrome [24]. They noted that both a 2 min walk test and a $75 \mathrm{~m}$ walk test yielded similar and reliable results. Based on Horemans et al.'s data, the $75 \mathrm{~m}$ SSWS and FPWS were chosen for this study [24]. This decision was substantiated by Simonsick et al.'s study, in which they reported that a distance-based test (versus a time-based test) increased participants' motivation to achieve maximum capacity in a sample of healthy, geriatric subjects [25].

\section{Fastest Possible Walking Speed: 38 m on Uneven Terrain}

The course consisted of trimmed grass, rocks, and generally uneven sandy terrain. The need to safely ambulate on uneven ground or other prevailing terrain is a critical criterion for justifying componentry [39]. A target distance of $38 \mathrm{~m}$ was selected for reasons similar to those described previously for the $75 \mathrm{~m}$ SSWS and FPWS tests.

\section{Fastest Possible Walking Speed: $6 \mathrm{~m}$ on Even Terrain}

We performed this test for several reasons. First, as previously established, a distance-based test is preferred to a time-based test because distance-based tests provide the benefit of a target goal. Second, at a distance of $6 \mathrm{~m}$, gait initiation and termination occur in proximity, placing primary emphasis on these more complex aspects of gait. Numerous studies have demonstrated that in the presence of neuromusculoskeletal pathology, changes in motor planning, step length, push-off force, and other parameters 
adversely affect the initiation and termination of gait [2638,41-43]. Finally, $6 \mathrm{~m}$ is the approximate distance of two lanes of traffic. All persons must be able to perform in a fight or flight situation [44-45]. Participants were instructed to "Pretend you are getting out the way of a bus.” The $6 \mathrm{~m}$ FPWS test puts both challenging points of gait, initiation and termination, very close to one another.

\section{Stair Descent Test}

A claimed benefit of the C-Leg is patients' ability to descend stairs in a step-over-step pattern versus most NMKMs for which leading descent with the prosthesis ("tap-step pattern") is recommended. Currently, no instruments have been validated for TF amputees relative to stair descent. We selected the MRPP to evaluate stair descent because it considers a step-over-step pattern, cueing (verbal, tactile, and stabilizing), rate of descent, and errors in foot placement of the leading leg ("scuff"). The MRPP considers these four elements and culminates them into one calculation, yielding a Performance Composite Score (PCS). The PCS is a numeric quantification that objectively measures functional stair descent.

A step-over-step pattern at a "normal" rate (1 step/ second with no errors) leads to a PCS of 0.77. Scores $<0.77$ are typically the result of performances that are either slower than $1 \mathrm{step} / \mathrm{second}$ or that contained errors. Scores $>0.77$ are typically faster than 1 step/second and do not contain errors [21].

If able, subjects were asked to walk down the laboratory staircase in a step-over-step pattern. However, if they were unable to do so, they were instructed to descend using their preferred method. The laboratory staircase consisted of six stairs $(17 \mathrm{~cm}$ high, $28 \mathrm{~cm}$ long, and $91 \mathrm{~cm}$ wide) with a handrail on both sides.

\section{Knee Preference}

In an effort to capture true patient preference and exclude a possible novelty effect, we asked subjects which knee mechanism they preferred and would actually want to continue using as the final evaluative measure. This measure was used to identify true subject preference regardless of the performance data.

\section{Protocol}

As a condition of enrollment, all subjects had to have self-reported $\geq 1$ episode of PT on their previously accommodated NMKM before the study. Once enrolled, subjects had to continue using their current NMKM for 90 days, during which time they were seen as needed for adjust- ments to swing and stance controls and alignment. Following this 90-day period, pretesting was administered by the study's licensed certified prosthetist while all subjects used their current accommodated NMKM. Pretesting included all of the evaluative measures previously described except for the question of knee preference, which was asked during posttesting so that subjects would have experience with both their current NMKM and a C-Leg.

The C-Legs were fitted, aligned, and adjusted according to the manufacturer's specifications and with the subjects' input. Swing and stance controls were adjusted with respect to patient preference and performance. The function of each subject's accommodated NMKM foot had to be matched with a prosthetic foot from the manufacturer's restrictive list of feet approved for use with the C-Leg. The prosthetist chose a foot that was functionally familiar for each subject, e.g., an energy-storing foot, a foot with an ankle unit, or both. Therefore the Otto Bock C-Walk ${ }^{\circledR}$ model 1C40 foot was chosen as the control foot unless subjects were $>220 \mathrm{lb}$ or an issue of clearance was present, in which case an Otto Bock LuXon ${ }^{\circledR}$ Max foot was used.

Follow-up visits were scheduled for 1 and 3 weeks post-C-Leg fitting, and subjects were seen for adjustments to swing and stance controls and alignment as deemed necessary by the prosthetist and with subjects' input. On two occasions (1) the same day that subjects were fitted with the C-Leg and (2) the day of the 1 week follow-up visit, subjects received preliminary gait training with the C-Leg from the prosthetist. Because of medical necessity, a physician prescribed PT in six cases (Table 2). Subjects accommodated with the C-Leg for 90 days after fitting.

Following the accommodation period with the C-Leg, posttesting was administered by the same licensed certified prosthetist who administered pretesting. Posttesting included the same battery of evaluative measures in the same order for every patient. At posttesting, the question of knee preference was asked for a total of nine measures instead of the eight administered at pretesting.

We used descriptive statistics to analyze two of the nine evaluative measures: participants' ability to descend stairs and their knee preference. We used paired $t$-tests to analyze the remaining seven evaluative measures: PEQ scores, self-reported number of stumbles, self-reported number of falls, $75 \mathrm{~m}$ SSWS, $75 \mathrm{~m}$ FPWS, $38 \mathrm{~m}$ FPWS (uneven terrain), and $6 \mathrm{~m}$ FPWS. We performed univariate analyses because of the small sample size. Because seven $t$-tests were performed (four for the walking tests and one each for the PEQ, stumbles, and falls), we 
Table 2.

Subject prosthesis information $(N=19$, mean \pm standard deviation age $=51 \pm 19 \mathrm{yr})$.

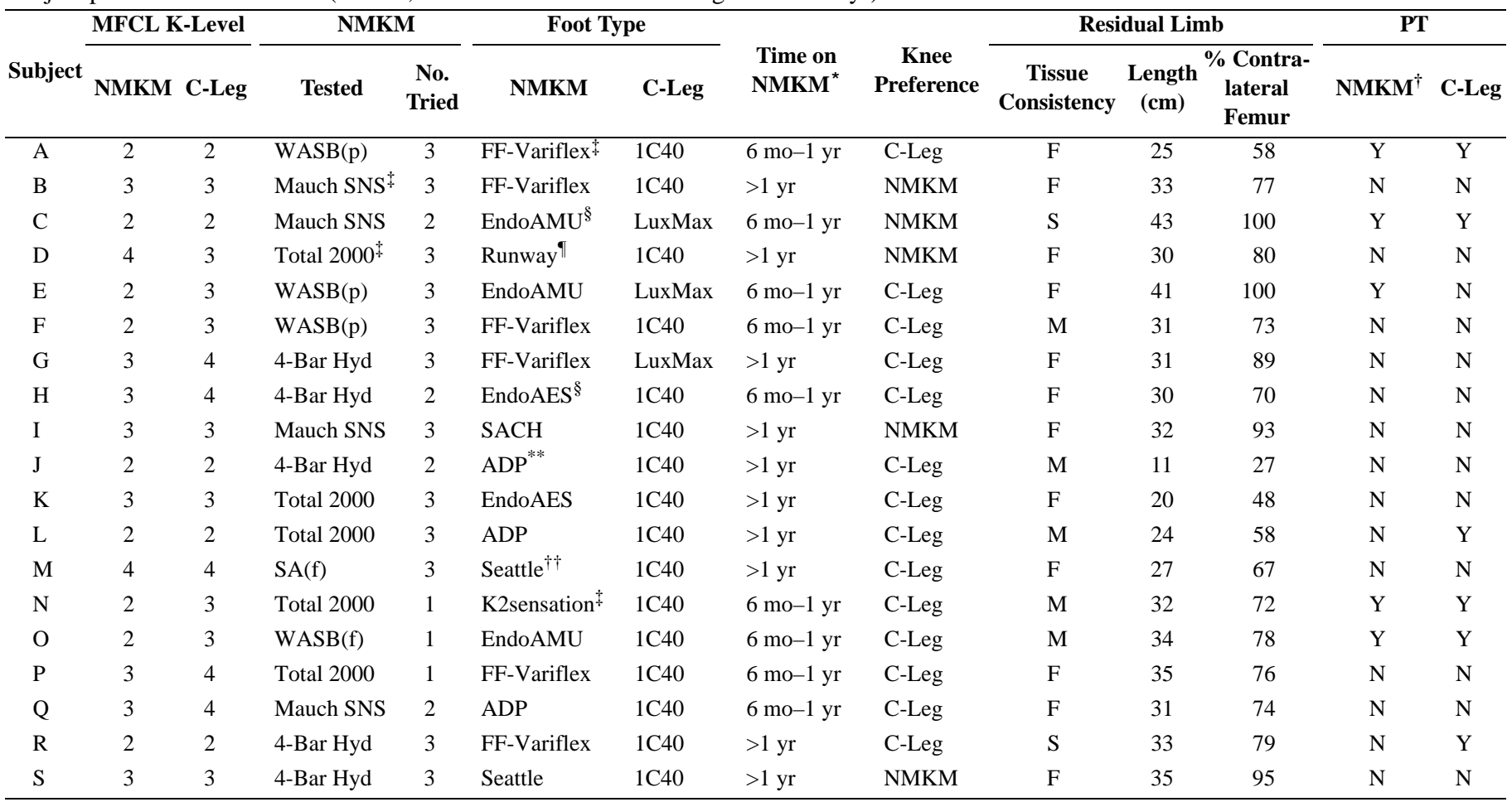

${ }^{*}$ Most participants wore numerous NMKMs and were tested on prosthetist/physician/subject preferred NMKM, which they wore for $>90$ days.

${ }^{\dagger}$ PT received during $90 \mathrm{~d}$ period between enrolling and pretesting.

${ }^{\ddagger}$ Ossur; Reykjavík, Iceland.

${ }^{\S}$ Endolite North America; Centerville, Ohio.

"Freedom Innovations Inc; Irvine, California.

** Otto Bock; Minneapolis, Minnesota.

${ }^{\dagger \dagger}$ Seattle Systems, Inc; Poulsbo, Washington.

1C40 = C-Walk ${ }^{\circledR}$ model 1C40, 4-Bar Hyd = 4-bar multiaxial knee joint with hydraulic swing-phase control, ADP = Advantage dynamic pylon, EndoAES = Endolite ankle with energy-storing foot, EndoAMU $=$ Endolite ankle with multiaxial foot, F $=$ firm, FF-Variflex $=$ Flex-Foot ${ }^{\circledR}$ Vari-Flex $^{\circledR}$, K2sensation $=$ K2 Sensa$\operatorname{tion}^{\mathrm{TM}}$, LuxMax $=\operatorname{LuXon}{ }^{\circledR}$ Max, $\mathrm{M}=$ medium, MFCL $=$ Medicare Functional Classification Level, $\mathrm{N}=$ no, NMKM = nonmicroprocessor knee mechanism, PT = physical therapy, $\mathrm{S}=$ soft, $\mathrm{SA}(\mathrm{f})=$ single axis friction, $\mathrm{SACH}=$ solid ankle cushion heel, Seattle = Seattle Lite Foot, Total $2000=$ Total Knee ${ }^{\circledR}$ model 2000 , WASB(f) = weight-activated stance-phase brake mechanism with friction swing-phase control, WASB(p) = weight-activated stance-phase brake mechanism with pneumatic swing-phase control, $\mathrm{Y}=$ yes.

performed a Bonferroni correction: the experiment-wide was $\alpha$ set at 0.10 and the individual test $\alpha$ at 0.01 . We used SAS ${ }^{\circledR}$ version 9.0 (SAS Institute Inc; Cary, North Carolina) for all analyses.

\section{RESULTS}

Of the 21 subjects enrolled, 19 completed the study (Table 1). One subject required an interface change because of fit problems and was thus excluded; the other subject was lost to follow-up. These two subjects were not included in the analysis.

\section{Prosthesis Evaluation Questionnaire}

We found a 20 percent improvement in PEQ scores when subjects used the C-Leg $(p=0.007)$ (Table 3). Figure 1 displays the difference in mean PEQ scores for each question for the C-Leg versus an NMKM.

\section{Stumbles and Falls}

Subjects using the NMKM self-reported an average of seven stumbles compared with an average of three stumbles when using the C-Leg, $(p=0.006)$ (Table 3). Subjects using the NMKM self-reported an average of three falls compared with an average of one fall when using the C-Leg $(p=0.03)$ (Table 3). 
Table 3.

Results of $t$-tests (mean \pm standard deviation) and percent change on performance evaluative measures for C-Leg vs nonmicroprocessor knee mechanism (NMKM) $(N=19)$.

\begin{tabular}{lcccc}
\hline \multicolumn{1}{c}{ Measure } & NMKM & C-Leg & $\boldsymbol{p}$-Value & \% Change \\
\hline PEQ & $942.3 \pm 269.3$ & $1,184.1 \pm 243.1$ & $0.007^{*}$ & 20 \\
Stumbles (No.) & $7 \pm 6$ & $3 \pm 4$ & $0.006^{*}$ & 59 \\
Falls (No.) & $3 \pm 3$ & $1 \pm 2$ & $0.03^{\dagger}$ & 64 \\
SSWS 75 m (s) & $101.3 \pm 47.8$ & $86.4 \pm 32.8$ & $0.03^{\dagger}$ & 15 \\
FPWS 75 m (s) & $81.4 \pm 33.6$ & $71.2 \pm 26.1$ & $0.005^{*}$ & 12 \\
FPWS 38 m Uneven Terrain (s) & $55.9 \pm 22.0$ & $44.2 \pm 16.4$ & $<0.001^{*}$ & 21 \\
FPWS 6 m (s) & $6.5 \pm 2.6$ & $5.4 \pm 2.2$ & $0.001^{*}$ & 17
\end{tabular}

*Significant at $p \leq 0.01$.

${ }^{\dagger}$ Significant at $p \leq 0.05$.

PEQ = Prosthesis Evaluation Questionnaire, SSWS = self-selected walking speed, FPWS = fastest possible walking speed

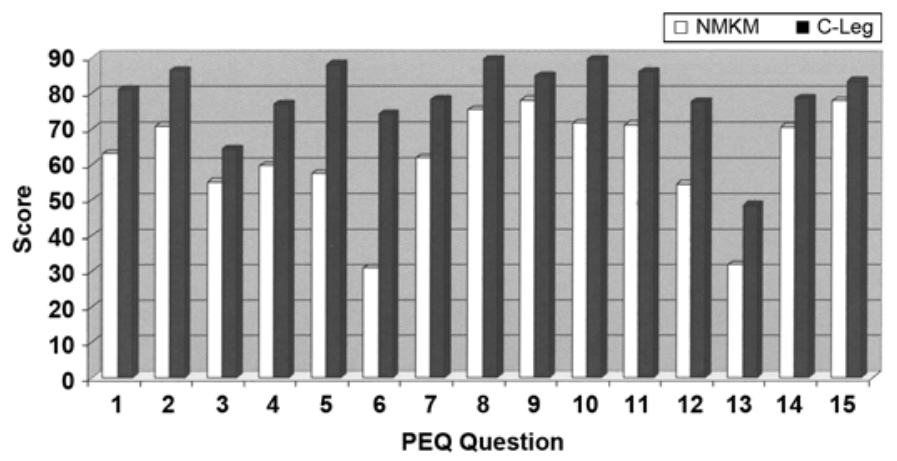

Figure 1.

Average responses of 19 subjects on first 15 questions of Prosthesis Evaluation Questionnaire (PEQ) for microprocessor knee mechanisms (i.e., C-Leg) vs nonmicroprocessor knee mechanisms (NMKMs).

\section{Walking Tests}

After a Bonferroni correction, all four of the "fastest" walking performance results remained significant at the individual test $\alpha$ of 0.01 (Table 3). For the walking tests, the percent difference in time required to complete the distance ranged from a 12 percent decrease (75 m FPWS) to a 21 percent decrease (38 m uneven terrain FPWS) (Tables 3 and 4). A decrease in time indicates an increase in walking speed.

An experiment-wide $\alpha$ of 0.10 means that 1 of 10 results would be significant by chance. Our findings of significance for five of the seven total $t$-tests and three of the four walking performance $t$-tests indicate that an intervention effect occurred that was beyond chance.

\section{Stair Descent}

Descriptive analyses of MRPP data revealed that 12 subjects (63\%) improved their PCS while wearing the
C-Leg. Two subjects improved their PCS while wearing the NMKM. Five subjects showed no change in their PCS (Figure 2). As defined by Pelland and McKinley, the ideal stair descent is 1 step/second with no taps, scuffs, or cues [21].

\section{Knee Preference}

At posttesting, when asked if given the option would participants prefer to keep the C-Leg or return to their previous NMKM, 14 participants (74\%) responded that they would like to continue to use the C-Leg and 5 (26\%) responded that they would rather return to using their previous NMKM. Of the five subjects who preferred to return to their previous NMKM, four had used their respective NMKM (Mauch SNS, Total Knee ${ }^{\circledR}$ [Ossur], or 4-bar hydraulic knee unit) for at least 10 years. Three of these four subjects were female and born with a congenital limb deficiency. Two of these rejected the C-Leg for cosmetic reasons, including an inability to readily accommodate heel height changes because of the limited choice of feet recommended by Otto Bock. The third subject born with congenital limb deficiency who rejected the C-Leg chose her previous NMKM because she felt it gave her more voluntary knee control. Two participants rejected the C-Leg because it was too expensive, even though they could have received the C-Leg at no out-of-pocket cost.

\section{DISCUSSION}

Using nine outcome measures (seven inferential and two descriptive), we found that TF amputees demonstrated increased performance while using the C-Leg. Most subjects, 74 percent, preferred the C-Leg. Rejection 
Table 4.

Results of eight performance evaluative measures, including preference, for all subjects (A-S, $N=19$ ).

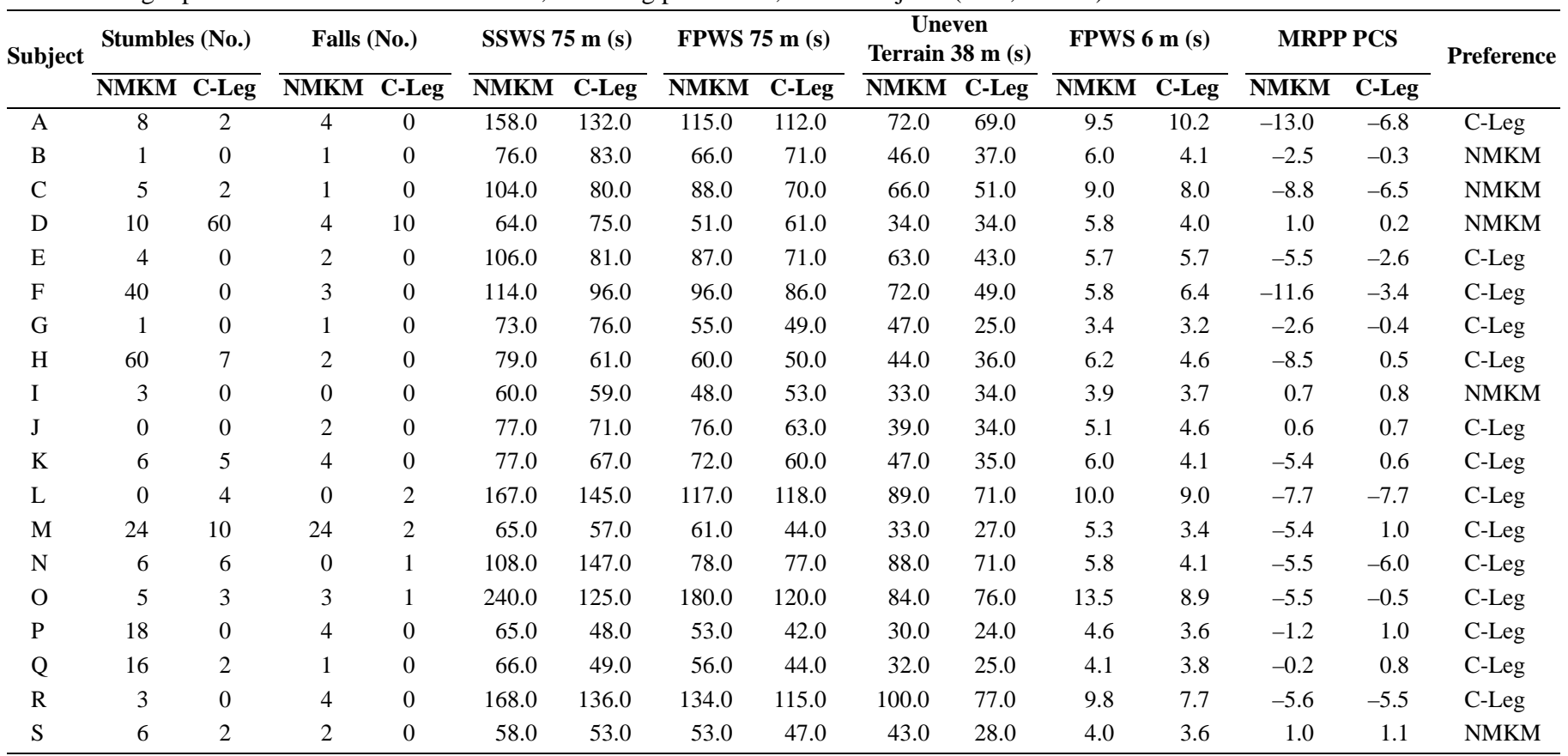

FPWS $=$ fastest possible walking speed, MRPP PCS $=$ Montreal Rehabilitation Performance Profile Performance Composite Score, NMKM = nonmicroprocessor knee mechanism, SSWS = self-selected walking speed.

of the C-Leg was predominately based on personal beliefs regarding either finance or cosmetics; the evaluative measures used in this study did not account for these two factors. Therefore, in clinical practice, early compo-

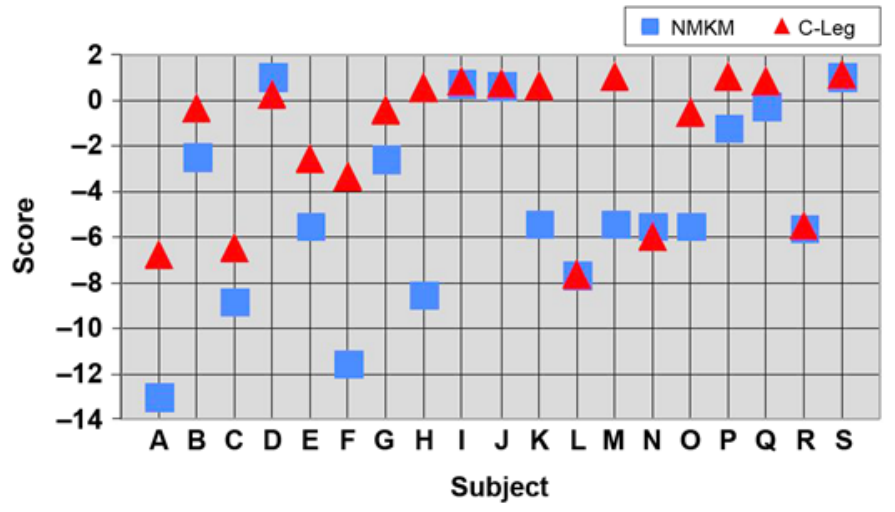

Figure 2.

Montreal Rehabilitation Performance Profile Performance Composite Scores (PCSs) of 19 subjects for C-Leg vs nonmicroprocessor knee mechanism (NMKM): 12 subjects improved PCS on C-Leg, 2 subjects improved PCS on NMKM, and 5 subjects showed no change. Ideal stair descent is 1 step/second with no taps, scuffs, or cues and yields ideal PCS of 0.77. Larger positive number means faster stair descent. Larger negative number means subject was slower than 1 step/second and/or had tap, scuff, or cue errors. nent selection should consider patient preference and values because they can be more important to some amputees than performance.

Clinicians must also consider that the CMS MFCLs are developed for reimbursement of prosthetic devices and based upon an amputee's actual or potential ability to ambulate within the community [39]. Currently, CMS requires TF amputees seeking reimbursement for any fluid NMKM and any MKM to be at the K3 ambulatory level. The K3 ambulator is described as a community ambulator who has the ability or potential to traverse most environmental barriers and may have prosthetic demands beyond simple locomotion. In comparison, the $\mathrm{K} 2$ ambulator is described as a limited community ambulator who has the ability or potential to traverse low-level environmental barriers. We conducted a secondary analysis that yielded an interesting finding. Using only the strict MFCL performance ratings typical in the clinical setting, the clinician in this study initially rated 9 of the 19 subjects as MFCL K2; these subjects would not have been considered MKM candidates. Of these nine subjects, four (47\%) increased their MFCL from K2 to K3; thus, amputees who according to MFCL guidelines would not have been candidates for the C-Leg actually improved their functional 
performance when using the C-Leg. Specifically, we found that not only are limited community G1 (MFCL K2) candidates for MKM technology, they are also able to advance to the MFCL K3 level when prescribed an MKM. In summary, our findings support that costly high-tech components may be under-prescribed [46]. This finding is exemplary of why amputees should be provided the opportunity to use the most advanced technology that could potentially increase their function.

Specific functional outcomes that improved with use of the C-Leg include (1) prosthesis function and prosthesis-related quality of life (as measured by the PEQ), with the most positive changes noted in ambulation on slippery surfaces and in tight spaces; (2) walking speed on a variety of surfaces; (3) self-reported number of stumbles and falls; and (4) stair descent. We used the PEQ to measure prosthesis function and prosthesis-related quality of life and self-report to count stumbles and falls. Hafner et al. created a new PEQ subsection to track stumbles and falls that we were unable to use because it had not been published at the time of this study [47]. Similar to our findings, Hafner et al. reported a significant decrease in stumbles and falls using their new PEQ subsection. Both our PEQ and our stumble and fall results compare favorably with those of Hafner et al. [47].

Orendurff et al. found stumble recovery was spontaneously reported as a favorite feature of the C-Leg [9]. Additionally, subjects preferred the C-Leg because of its capability to adapt to any walking speed [9]. Orendurff et al. speculated that the C-Leg instilled increased confidence, which in turn decreased stumbles and falls and increased users' ability to vary their walking speed [9]. Thus, TF amputees may increase their walking speed once they have gained sufficient confidence using the C-Leg because they experience substantially fewer stumbles and falls.

Recent studies note that subjects using a C-Leg versus a previously accommodated NMKM tend to have a faster SSWS [9,47]. Orendurff et al. collected metabolic data at three predetermined walking speeds $(0.8,1.0$, and $1.3 \mathrm{~m} / \mathrm{s}$ ) and one SSWS while subjects walked over level terrain. We expanded upon the findings of Orendurff et al. by evaluating walking speed under four different conditions to determine if walking speed would increase when subjects used the C-Leg [9]. We found a significant decrease in the time required to complete each of the four walking conditions (an increase in speed) when subjects used the C-Leg.
Uneven terrain is an unpredictable surface that requires the amputee's constant attention to foot placement and knee stability. Accordingly, increased walking speed might be least expected on uneven terrain. However, we found that walking speed on uneven terrain did increase. When using the C-Leg on uneven terrain, subjects showed the greatest decrease in time required to cover the given distance $(21 \%, p<0.001)$. Features of the C-Leg such as stumble recovery may yield an increase in user confidence that enables an increase in speed on prevailing terrain.

Walking speed is commonly defined as the time required to walk a given distance, whereas cadence is commonly defined as the number of steps taken in a given time. Normally, as walking speed increases, cadence also increases; i.e., amputees who can change their walking speed can also vary their cadence. The ability to demonstrate variable walking speed and cadence demands more from a prosthesis than "simple" or "normal" locomotion. A simple friction knee without swing control cannot change cadence and speed. In contrast, fluid-control knees (especially MKMs) can change cadence and speed by adjusting to the user's immediate needs. Because of rapidly and continuously changing environmental conditions, community ambulation demands variable cadence from knee prostheses and their users. For example, one must avoid an oncoming vehicle when crossing the street. Acute changes in speed are also necessary to achieve performance levels for "recreational" activities related to cardiovascular health [44].

In their review of the literature, Van Iersal et al. found no optimal assessment of stair negotiation for amputees [20]. We used the MRPP as our measure of stair descent because even though the MRPP has not been proved valid or reliable in the amputee population, it does consider stair descent. Our interest in using the MRPP was to determine if the C-Leg would enable TF amputees to descend stairs using a step-over-step pattern in accordance with the manufacturer's claim. We found that 63 percent of our sample was able to descend stairs in this manner while using the C-Leg. However, this result does not mean that the stepover-step pattern is the preferred, safest, or most efficient stair descent method. In future studies, we will ask subjects to descend stairs in their preferred manner and then ask subjects to descend with the step-over-step pattern if they can. Results can then be compared.

The MRPP PCS is based on the premise that faster is better; a more positive score translates to a faster than 
normal stair descent (1 step/second). However, faster may not always be better, more energy efficient, or safer, as one could surely fall or leap down a set of stairs faster than descending on two feet. The C-Leg did allow many subjects to descend stairs using a step-over-step pattern under controlled conditions, although it may not have been their preferred method. Future studies will also investigate the effects of various knee mechanisms on stair ascent and ramp descent/ascent.

This study had several limitations. First, only one rater was included. While one rater eliminates interrater bias, the potential for intervention bias is introduced. In accordance with the design and clinical nature of this study and for safety reasons, the one rater was not blinded. Second, the sample size of 19 limited the analyses that could be performed; i.e., the sample size was insufficient for models that considered potential confounding factors such as time since amputation, residual limb length, age, and subjects' functional levels. The sample size was adequate for analysis of this initial pilot data. The rigor of the study could have been increased by limiting subjects to a single NMKM, e.g., Mauch SNS, but increasing rigor would also decrease power by further limiting the number of subjects.

One additional benefit of this study is that we tested a protocol of evaluative measures that prosthetic practitioners can easily repeat in the clinic. The protocol used in this study is important for providing the practitioner an organized framework for gathering clinical data. These data are (1) clinically vital for determining patient functional outcomes with one specific component compared with another and (2) essential to the process of justifying medical necessity, patient need, and patient preference. Based on these findings, a similar protocol is planned for use in future studies that investigate candidacy and medical necessity of MKM technology in a more functionally diverse population of TF amputees.

\section{CONCLUSIONS}

This study compared performance of subjects using a wide variety of NMKMs and the C-Leg. Using the C-Leg, most subjects demonstrated greater prosthesis function and prosthesis-related quality of life, decreased selfreported falls and stumbles, increased walking speed under several conditions, and improved stair descent. In addition, most subjects preferred the C-Leg.

\section{ACKNOWLEDGMENTS}

We would like to thank the ethics review committee members and Westcoast Brace and Limb (Tampa, Florida) for assembling the ethics review committee, which comprised physicians, orthotists, prosthetists, therapists, component suppliers, patients, and laypersons and ensured that the study protocol (1) provided clearly defined end points, (2) would not expose subjects to risks greater than those in traditional care, and (3) clarified that subjects could withdraw from the study without repercussion at any point.

This material was based on work supported by the U.S. Department of Education, Rehabilitation Service Administration (project 6140100000/award H235J050020) "Demonstration project on prosthetics and orthotics.”

The authors have declared that no competing interests exist.

\section{REFERENCES}

1. Hafner BJ. Perceptive evaluation of prosthetic foot and ankle systems. J Prosthet Orthot. 2005;17(4):S42-46.

2. Legro MW, Reiber GD, Smith DG, Del Aguila M, Larsen J, Boone D. Prosthesis Evaluation Questionnaire for persons with lower limb amputations: Assessing prosthesis-related quality of life. Arch Phys Med Rehabil. 1998;79(8):931-38. [PMID: 9710165]

3. Boone D, Coleman KL. Use of the Prosthesis Evaluation Questionnaire (PEQ). J Prosthet Orthot. 2006;18(1 Suppl): P68-79.

4. Miller WC, Speechley M, Deathe B. The prevalence and risk factors of falling and fear of falling among lower extremity amputees. Arch Phys Med Rehabil. 2001;82(8): 1031-37. [PMID: 11494181]

5. Mackenzie L, Byles J, D’Este C. Validation of self-reported fall events in intervention studies. Clin Rehabil. 2006; 20(4):331-39. [PMID: 16719031]

6. Jenkins P, Earle-Richardson G, Slingerland DT, May J. Time dependent memory decay. Am J Ind Med. 2002;41(2):98-101. [PMID: 11813214]

7. Peel N. Validating recall of falls by older people. Accid Anal Prev. 2000;32(3):371-72. [PMID: 10776852]

8. Schmalz T, Blumentritt S, Jarasch R. Energy expenditure and biomechanical characteristics of lower limb amputee gait: The influence of prosthetic alignment and different prosthetic components. Gait Posture. 2002;16(3):255-63. [PMID: 12443950]

9. Orendurff MS, Segal AD, Klute GK, McDowell ML, Pecoraro JA, Czerniecki JM. Gait efficiency using the C-Leg. J Rehabil Res Dev. 2006;43(2):239-46. [PMID: 16847790] 
10. Johansson JL, Sherrill DM, Riley PO, Bonato P, Herr H. A clinical comparison of variable-damping and mechanically passive prosthetic knee devices. Am J Phys Med Rehabil. 2005;84(8):563-75. [PMID: 16034225]

11. Perry J, Burnfield JM, Newsam CJ, Conley P. Energy expenditure and gait characteristics of a bilateral amputee walking with C-Leg prostheses compared with stubby and conventional articulating prostheses. Arch Phys Med Rehabil. 2004;85(10):1711-17. [PMID: 15468036]

12. Stinus H. Biomechanics and evaluation of the microprocessorcontrolled C-Leg exoprosthesis knee joint [German]. Z Orthop Ihre Grenzgeb. 2000;138(3):278-82. [PMID: 10929622]

13. Datta D, Heller B, Howitt J. A comparative evaluation of oxygen consumption and gait pattern in amputees using Intelligent Prostheses and conventionally damped knee swing-phase control. Clin Rehabil. 2005;19(4):398-403. [PMID: 15929508]

14. Buckley JG, Spence WD, Solomonidis SE. Energy cost of walking: Comparison of "intelligent prosthesis" with conventional mechanism. Arch Phys Med Rehabil. 1997;78(3): 330-33. [PMID: 9084360]

15. Taylor MB, Clark E, Offord EA, Baxter C. A comparison of energy expenditure by a high level trans-femoral amputee using the Intelligent Prosthesis and conventionally damped prosthetic limbs. Prosth Orthot Int. 1996;20(2): 116-21. [PMID: 8876005]

16. Jones SF, Twigg PC, Scally AJ, Buckley JG. The mechanics of landing when stepping down in unilateral lower-limb amputees. Clin Biomech (Bristol, Avon). 2006;21(2):184-93. [PMID: 16274904]

17. Schmalz T, Blumentritt S, Marx B. Biomechanical analysis of stair ambulation in lower limb amputees. Gait Posture. 2007;25(2):267-78. [PMID: 16725325]

18. Hamel KA, Cavanagh PR. Stair performance in people aged 75 and older. J Am Geriatr Soc. 2004;52(4):563-67. [PMID: 15066071]

19. Ayis S, Gooberman-Hill R, Bowling A, Ebrahim S. Predicting catastrophic decline in mobility among older people. Age Ageing. 2006;35(4):382-87. [PMID: 16638760]

20. Van Iersel MB, Olde Rikkert MG, Mulley GP. Is stair negotiation measured appropriately in functional assessment scales? Clin Rehabil. 2003;17(3):325-33.

[PMID: 12735541$]$

21. Pelland L, McKinley P. The Montreal Rehabilitation Performance Profile: A task-analysis approach to quantify stair descent performance in children with intellectual disability. Arch Phys Med Rehabil. 2001;82(8):1106-14. [PMID: 11494191]

22. Montero-Odasso M, Schapira M, Varela C, Pitteri C, Soriano ER, Kaplan R, Camera LA, Mayorga LM. Gait velocity in senior people. An easy test for detecting mobility impairment in community elderly. J Nutr Health Aging. 2004;8(5):340-43. [PMID: 15359349]
23. Montero-Odasso M, Schapira M, Soriano ER, Varela M, Kaplan R, Camera LA, Mayorga LM. Gait velocity as a single predictor of adverse events in healthy seniors aged 75 years and older. J Gerontol A Biol Sci Med Sci. 2005; 60(10):1304-9. [PMID: 16282564]

24. Horemans HL, Beelen A, Nollet F, Lankhorst GJ. Reproducibility of walking at self-preferred and maximal speed in patients with postpoliomyelitis syndrome. Arch Phys Med Rehabil. 2004;85(12):1929-32. [PMID: 15605328]

25. Simonsick EM, Montgomery PS, Newman AB, Bauer DC, Harris T. Measuring fitness in healthy older adults: The Health ABC Long Distance Corridor Walk. J Am Geriatr Soc. 2001;49(11):1544-48. [PMID: 11890597]

26. Jones SF, Twigg PC, Scally AJ, Buckley JG. The gait initiation process in unilateral lower-limb amputees when stepping up and stepping down to a new level. Clin Biomech (Bristol, Avon). 2005;20(4):405-13. [PMID: 15737448]

27. Viton JM, Timsit M, Mesure S, Massion J, Franceschi JP, Delarque A. Asymmetry of gait initiation in patients with unilateral knee arthritis. Arch Phys Med Rehabil. 2000; 81(2):194-200. [PMID: 10668774]

28. Polcyn AF, Lipsitz LA, Kerrigan DC, Collins JJ. Agerelated changes in the initiation of gait: Degradation of central mechanisms for momentum generation. Arch Phys Med Rehabil. 1998;79(12):1582-89. [PMID: 9862305]

29. DeVita P. The selection of a standard convention for analyzing gait data based on the analysis of relevant biomechanical factors. J Biomech. 1994;27(4):501-8. [PMID: 8188730]

30. Michel V, Chong RK. The strategies to regulate and to modulate the propulsive forces during gait initiation in lower limb amputees. Exp Brain Res. 2004;158(3):356-65. [PMID: 15167976]

31. Michel V, Do MC. Are stance ankle plantar flexor muscles necessary to generate propulsive force during human gait initiation? Neurosci Lett. 2002;325(2):139-43.

[PMID: 12044640]

32. Rossi SA, Doyle W, Skinner HB. Gait initiation of persons with below-knee amputation: The characterization and comparison of force profiles. J Rehabil Res Dev. 1995;32(2): 120-27. [PMID: 7562651]

33. Pinzur MS, Asselmeier M, Smith D. Dynamic electromyography in active and limited walking below-knee amputees. Orthopedics. 1991;14(5):535-37; discussion 537-38. [PMID: 2062730]

34. Jaeger RJ, Vanitchatchavan P. Ground reaction forces during termination of human gait. J Biomech. 1992;25(10): 1233-36. [PMID: 1400524]

35. Wearing SC, Urry S, Smeathers JE, Battistutta D. A comparison of gait initiation and termination methods for obtaining plantar foot pressures. Gait Posture. 1999;10(3): 255-63. [PMID: 10567758] 
36. Meier MR, Desrosiers J, Bourassa P, Blaszczyk J. Effect of type II diabetic peripheral neuropathy on gait termination in the elderly. Diabetologia. 2001;44(5):585-92. [PMID: 11380076]

37. Perry SD, Santos LC, Patla AE. Contribution of vision and cutaneous sensation to the control of centre of mass (COM) during gait termination. Brain Res. 2001;913(1):27-34. [PMID: 11532244]

38. Tirosh O, Sparrow WA. Age and walking speed effects on muscle recruitment in gait termination. Gait Posture. 2005; 21(3):279-88. [PMID: 15760743]

39. Centers for Medicare and Medicaid Services. U.S. Department of Health and Human Services. Healthcare Common Procedure Coding System. Springfield (VA): U.S. Department of Commerce, National Technical Information Service; 2007.

40. Prosthetic Forum Summary Report. Walter Reed Army Medical Center C-Leg Microprocessor Knee Patient Evaluation Protocol 2003. J Prosthet Orthot. 2004;16(1):31-39.

41. Cao C, Ashton-Miller JA, Schultz AB, Alexander NB. Abilities to turn suddenly while walking: Effects of age, gender, and available response time. J Gerontol A Biol Sci Med Sci. 1997;52(2):M88-93. [PMID: 9060975]
42. Stein RB, Hase K. Stopping and turning during human walking. Prog Brain Res. 1999;123:445-53. [PMID: 10635740]

43. Imms FJ, Edholm OG. Studies of gait and mobility in the elderly. Age Ageing. 1981;10(3):147-56. [PMID: 7270322$]$

44. Gailey RS. Recreational pursuits for elders with amputations. Top Geriatr Rehabil. 1992;8(1):39-58.

45. Kegel B, Webster JC, Burgess EM. Recreational activities of lower extremity amputees: A survey. Arch Phys Med Rehabil. 1980;61(6):258-64. [PMID: 7377953]

46. Gailey RS, Roach KE, Applegate EB, Cho B, Cunniffe B, Licht S, Maguire M, Nash MS. The amputee mobility predictor: An instrument to assess determinants of the lowerlimb amputee's ability to ambulate. Arch Phys Med Rehabil. 2002;83(5):613-27. [PMID: 11994800]

47. Hafner BJ, Willingham LL, Buell NC, Allyn KJ, Smith DG. Evaluation of function, performance, and preference as transfemoral amputees transition from mechanical to microprocessor control of the prosthetic knee. Arch Phys Med Rehabil. 2007;88(2):207-17. [PMID: 17270519] Erratum in Arch Phys Med Rehabil. 2007;88(4):544.

Submitted for publication April 4, 2007. Accepted in revised form August 30, 2007. 